\title{
Formal Synthesis of (-)-Perhydrohistrionicotoxin via Cyclic Amino Acid Ester-Enolate Claisen Rearrangement and Ring- Closing Metathesis
}

Sanghee Kim,* Hyojin Ko, Taeho Lee and Deukjoon Kim

\section{Contents}

General Experimatal Method

Experimental procedures for the synthesis of $\mathbf{9}$ and other known advanced intermediates (16 and 17)

S3-S5

Comparison of the spectral data

(formic acid 7-butyl-2-oxo-1-aza-spiro[5.5] undec-8-yl Ester (16))

Comparison of the spectral data

((-)-depentylperhydrohistrionicotoxin (17))

${ }^{1} \mathrm{H}$ NMR and ${ }^{13} \mathrm{C}$ NMR spectra of compound 7

${ }^{1} \mathrm{H}$ NMR and ${ }^{13} \mathrm{C}$ NMR spectra of compound $\mathbf{1 0}$

S10-S11

${ }^{1} \mathrm{H}$ NMR and ${ }^{13} \mathrm{C}$ NMR spectra of compound 13

S12-S13

${ }^{1} \mathrm{H}$ NMR and ${ }^{13} \mathrm{C}$ NMR spectra of compound 14

S14-S15

${ }^{1} \mathrm{H}$ NMR and ${ }^{13} \mathrm{C}$ NMR spectra of compound 4

S16-S17

${ }^{1} \mathrm{H}$ NMR and ${ }^{13} \mathrm{C}$ NMR spectra of compound $\mathbf{1 5}$

S18-S19

${ }^{1} \mathrm{H}$ NMR spectrum of mixture of $\mathbf{1 5}$ and epi $\mathbf{1 5}$ (crude)

S20

${ }^{1} \mathrm{H}$ NMR and ${ }^{13} \mathrm{C}$ NMR spectra of compound $\mathbf{3}$

S21-S22

COSY, HSQC, and HMBC spectra of compound $\mathbf{3}$

$\mathrm{S} 23-\mathrm{S} 25$

${ }^{1} \mathrm{H}$ NMR and ${ }^{13} \mathrm{C}$ NMR spectra of compound $\mathbf{1 6}$

S26-S27

${ }^{1} \mathrm{H}$ NMR and ${ }^{13} \mathrm{C}$ NMR spectra of compound 17

S28-S29 
General. All chemicals were reagent grade and used as purchased. All reactions were performed under an inert atmosphere of dry argon or nitrogen using distilled dry solvents. Reactions were monitored by TLC analysis using silica gel 60 F-254 thin layer plates. Flash column chromatography was carried out on silica gel 60 (230-400 mesh). Melting points are uncorrected. IR spectra were measured on Fourier Transform Infrared spectrometer. Optical rotations were measured using sodium light (D line 589.3 $\mathrm{nm})$. Mass spectra (MS) were recorded at 70 or $30 \mathrm{eV}$ using electron impact (EI) or chemical ionization (CI). High resolution mass spectra (HRMS) were recorded using EI or CI. 
(S)-(E)-Oct-3-en-2-ol (9).

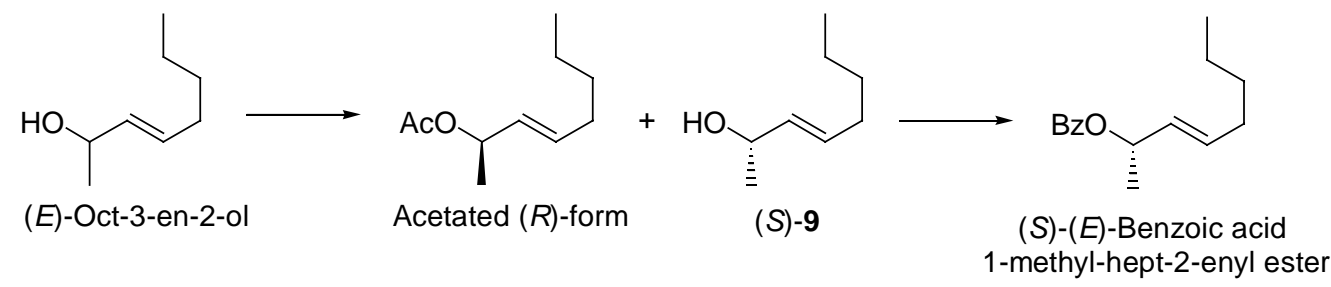

To a solution of $(E)$-oct-3-en-2-ol $(9.8 \mathrm{~g}, 76 \mathrm{mmol})$ in DIPE $(240 \mathrm{~mL})$ was added lipozyme (4.9 g, $50 \mathrm{wt} \%)$ and vinyl acetate $(24 \mathrm{~mL})$. The reaction mixture was stirred at $35{ }^{\circ} \mathrm{C}$ for $15 \mathrm{~h}$. The conversion was monitored by $\mathrm{GC}$ analysis (HP-1, detector temperature: $200{ }^{\circ} \mathrm{C}$, injector temperature: $280{ }^{\circ} \mathrm{C}$, oven temperature: $100{ }^{\circ} \mathrm{C}(10 \mathrm{~min})$ to $200{ }^{\circ} \mathrm{C}(10 \mathrm{~min}), 5{ }^{\circ} \mathrm{C} / \mathrm{min}$, retention time: $7.15 \mathrm{~min}(S)-9,8.52 \mathrm{~min}$ acetated $(R)$-form). The reaction mixture was cooled to room temperature and filtered through a Celite pad. The filtrate was evaporated under reduced pressure and purified by column chromatography on silica gel (petroleum ether/ether $=3: 1)$ to give $(S)-9(4.8 \mathrm{~g}, 38$ mmol, 49\%, $\left.[\alpha]_{\mathrm{D}}^{25}-12.3\left(c \mathrm{1.0}, \mathrm{CHCl}_{3}\right)\right)$ and acetated $(R)$-form $(6.5 \mathrm{~g}, 38 \mathrm{mmol}, 50 \%)$. The ${ }^{1} \mathrm{H}$ NMR spectral data of $(S)-9$ were identical with those reported. $(S)-9$ was converted to the corresponding benzoate, $(S)$ - $(E)$-benzoic acid 1-methyl-hept-2-enyl ester, for the determination of its enantiomeric purity by chiral HPLC $(98.5$ ee, $(R, R)$ Whelk-O1, 2\% isopropyl alcohol in hexane, flow rate: $1.0 \mathrm{~mL} / \mathrm{min}$, retention time: 4.49 $\min (S)-(E)$-benzoic acid 1-methyl-hept-2-enyl ester). 
(6S,7S,8S)-Formic acid 7-butyl-2-oxo-1-aza-spiro[5.5] undec-8-yl Ester (16)

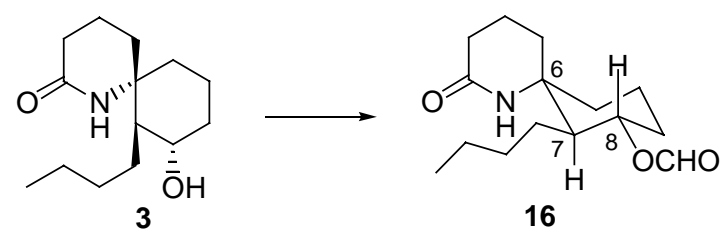

To a solution of $\mathbf{3}(11 \mathrm{mg}, 0.046 \mathrm{mmol})$ in $\mathrm{CH}_{2} \mathrm{Cl}_{2}(1 \mathrm{~mL})$ was added pyridine $(0.04$

$\mathrm{mL}, 0.48 \mathrm{mmol})$, followed by treatment with the mixture of formic acid $(0.01 \mathrm{~mL}, 0.26$

mmol $)$ and acetic anhydride $(0.02 \mathrm{~mL}, 0.24 \mathrm{mmol})$. After the reaction mixture was

stirred for $1 \mathrm{~h}$ at room temperature, it was concentrated and purified by silica gel column chromatography in EtOAc to give formate $16(11 \mathrm{mg}, 89 \%)$ as white solid. $\mathrm{Mp}$ 135-137 ${ }^{\circ} \mathrm{C}$; IR (film) $v_{\max } 2361.1,2341.8,1722.6,1658.9 \mathrm{~cm}^{-1} ;{ }^{1} \mathrm{H}$ NMR $\left(\mathrm{CDCl}_{3}, 300\right.$ MHz) $\delta 0.86(\mathrm{t}, J=7.2 \mathrm{~Hz}, 3 \mathrm{H}), 1.11-1.92(\mathrm{~m}, 17 \mathrm{H}), 2.17-2.80(\mathrm{~m}, 1 \mathrm{H}), 2.30-2.41(\mathrm{~m}$, 1H), $5.00\left(\mathrm{~m}, \mathrm{~W} 1 / 2=15.3 \mathrm{~Hz}, 1 \mathrm{H} ; \delta 4.81\left(\mathrm{ddd}, J=10.2,10.2,4.2 \mathrm{~Hz}\right.\right.$ at $\left.\left.\mathrm{C}_{6} \mathrm{D}_{6}\right)\right), 6.46$ (br s, $1 \mathrm{H}), 8.09$ (s, $1 \mathrm{H}) ;{ }^{13} \mathrm{C} \mathrm{NMR}\left(\mathrm{CDCl}_{3}, 75 \mathrm{MHz}\right) \delta 13.9,16.8,18.5,22.9,26.8,27.7$, 29.4, 31.2, 32.2, 36.1, 50.7, 58.6, 74.0, 160.3, 172.2; HRMS-EI (Calcd for $\mathrm{C}_{15} \mathrm{H}_{25} \mathrm{NO}_{3}$ ) 267.1834 , found 267.1833

\section{(-)-Depentylperhydrohistrionicotoxin (17)}

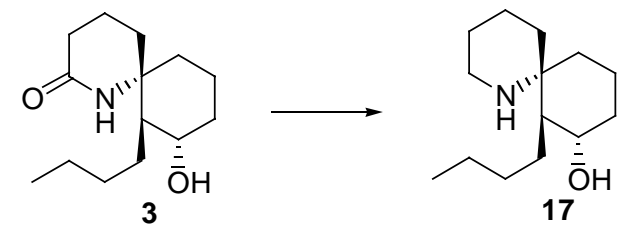


To a solution of 3 (13 mg, $0.054 \mathrm{mmol})$ in THF (3 mL) was added $\mathrm{LiAlH}_{4}(20 \mathrm{mg}$, $0.53 \mathrm{mmol}$ ) at $0{ }^{\circ} \mathrm{C}$. The mixture was heated to reflux for $5 \mathrm{~h}$ and cooled to room temperature. The reaction mixture was quenched with saturated aqueous $\mathrm{Na}_{2} \mathrm{SO}_{4}$ solution and extracted with $\mathrm{CH}_{2} \mathrm{Cl}_{2}$ three times. The combined organic layers were dried over $\mathrm{Na}_{2} \mathrm{SO}_{4}$ and concentrated. The crude product was purified by silica gel column chromatography $\left(\mathrm{CH}_{2} \mathrm{Cl}_{2} / \mathrm{MeOH}=3: 1\right)$ to give $17(10 \mathrm{mg}, 82 \%)$ as colorless oil. $[\alpha]^{21}$ $-43.7\left(c\right.$ 1.0, $\left.\mathrm{CHCl}_{3}\right)\left\{\right.$ lit. $\left.^{5 \mathrm{~d}}[\alpha]^{25}{ }_{\mathrm{D}}-45.3\left(c 7.8, \mathrm{CHCl}_{3}\right)\right\}$; IR (film) $v_{\max }$ 3271.2, 2361.1, $2341.8 \mathrm{~cm}^{-1} ;{ }^{1} \mathrm{H} \mathrm{NMR}\left(\mathrm{CDCl}_{3}, \mathrm{D}_{2} \mathrm{O}, 300 \mathrm{MHz}\right) \delta 0.89(\mathrm{t}, J=6.9 \mathrm{~Hz}, 3 \mathrm{H}), 1.0-2.0(\mathrm{~m}$, 21H), $2.78(\mathrm{~m}, 1 \mathrm{H}), 2.92(\mathrm{~m}, 1 \mathrm{H}), 3.87(\mathrm{dd}, J=5.4,2.7 \mathrm{~Hz}, 1 \mathrm{H}) ;{ }^{13} \mathrm{C} \mathrm{NMR}\left(\mathrm{CDCl}_{3}\right.$, $\left.\mathrm{D}_{2} \mathrm{O}, 75 \mathrm{MHz}\right) \delta 14.0,15.1,19.5,23.0,27.2,27.3,27.9,30.5,33.2,37.2,40.1,41.8$, 54.5, 69.9; HRMS-EI (Calcd for $\mathrm{C}_{14} \mathrm{H}_{27} \mathrm{NO}$ ) 225.2093, found 225.2092. 


\section{Comparison of the spectral data}

Formic acid 7-butyl-2-oxo-1-aza-spiro[5.5]undec-8-yl Ester (16)

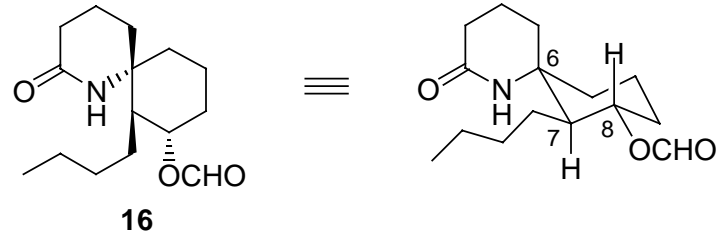

\begin{tabular}{|c|c|c|}
\hline${ }^{13} \mathrm{C} \mathrm{NMR}\left(\mathrm{CDCl}_{3}\right)$ & $171.9,160.1,58.5$ & Speckamp $1978^{5 \mathrm{c}}$ \\
\hline${ }^{1} \mathrm{H}$ NMR $\left(\mathrm{C}_{6} \mathrm{D}_{6}\right)$ & $\begin{array}{l}8.40(\mathrm{~s}, 1 \mathrm{H}), 7.68(\mathrm{~s}, 1 \mathrm{H}), 4.65-5.00(\mathrm{t} \text { of } \mathrm{d}, 1 \mathrm{H}), 0.80- \\
2.40(\mathrm{~m}, 22 \mathrm{H})\end{array}$ & Speckamp $1978^{5 c}$ \\
\hline $\begin{array}{c}\mathrm{H}_{8}{ }^{1} \mathrm{H} \text { NMR } \\
\left(\mathrm{C}_{6} \mathrm{D}_{6}, 300 \mathrm{MHz}\right)\end{array}$ & ddd, $J=10.5,10.5,4.5 \mathrm{~Hz}$ & Speckamp $1978^{5 c}$ \\
\hline $\mathrm{H}_{8}{ }^{1} \mathrm{H} \mathrm{NMR}\left(\mathrm{CDCl}_{3}\right)$ & $4.95(\mathrm{~m}, \mathrm{~W} 1 / 2=15.0 \mathrm{~Hz})$ & Evans $1979^{5 \mathrm{i}}$ \\
\hline $\begin{array}{c}{ }^{1} \mathrm{H} \mathrm{NMR} \\
\left(\mathrm{CDCl}_{3}, 90 \mathrm{MHz}\right)\end{array}$ & $\begin{array}{l}8.06(\mathrm{~s}, 1 \mathrm{H}), 6.89(\mathrm{~s}, 1 \mathrm{H}), 4.78-5.16(\mathrm{~m}, 1 \mathrm{H}), 2.10-2.44 \\
(\mathrm{~m}, 2 \mathrm{H}), 0.72-2.20(\mathrm{~m}, 20 \mathrm{H})\end{array}$ & Evans $1982^{\text {S1a }}$ \\
\hline $\begin{array}{c}{ }^{13} \mathrm{C} \mathrm{NMR} \\
\left(\mathrm{CDCl}_{3}, 22.5 \mathrm{MHz}\right)\end{array}$ & $\begin{array}{l}171.9,160.1,73.9,58.5,50.7,36.1,32.3,31.4,29.5 \\
27.8,26.7,23.0,18.5,16.9,13.9\end{array}$ & Evans $1982^{\text {Sla }}$ \\
\hline $\begin{array}{c}{ }^{1} \mathrm{H} \text { NMR } \\
\left(\mathrm{CDCl}_{3}, 300 \mathrm{MHz}\right)\end{array}$ & $\begin{array}{l}8.09(\mathrm{~s}, 1 \mathrm{H}), 6.50(\mathrm{~s}, 1 \mathrm{H}), 5.00(\mathrm{~m}, \mathrm{~W} 1 / 2=15.3 \mathrm{~Hz} \\
1 \mathrm{H}), 2.30-2.41(\mathrm{~m}, 1 \mathrm{H}), 2.17-2.80(\mathrm{~m}, 1 \mathrm{H}), 1.10-1.90 \\
(\mathrm{~m}, 17 \mathrm{H})\end{array}$ & $\begin{array}{l}\text { Our synthetic } \\
\text { compound }\end{array}$ \\
\hline $\begin{array}{c}\mathrm{H}_{8}{ }^{1} \mathrm{H} \text { NMR } \\
\left(\mathrm{C}_{6} \mathrm{D}_{6}, 300 \mathrm{MHz}\right)\end{array}$ & $4.81(\mathrm{ddd}, J=10.2,10.2,4.2 \mathrm{~Hz})$ & $\begin{array}{l}\text { Our synthetic } \\
\text { compound }\end{array}$ \\
\hline $\begin{array}{c}{ }^{13} \mathrm{C} \mathrm{NMR} \\
\left(\mathrm{CDCl}_{3}, 75 \mathrm{MHz}\right)\end{array}$ & $\begin{array}{l}172.2,160.3,74.0,58.6,50.7,36.132 .2,31.2,29.4, \\
27.7,26.8,22.9,18.5,16.8,13.9\end{array}$ & $\begin{array}{l}\text { Our synthetic } \\
\text { compound }\end{array}$ \\
\hline
\end{tabular}

\footnotetext{
S1 (a) Evans, D. A.; Thomas, E. W.; Cherpeck, R. E. J. Am. Chem. Soc. 1982, 104, 3695. (b) Pearson, A. J. J. Chem. Soc., Perkin Trans. I 1983, 1421. (c) Tanner, D.; Somfai, P. Tetrahedron, 1986, 42, 5657. (d) Carruthers, W.; Cumming, S. A. J. Chem. Soc., Perkin Trans. I 1983, 2383.
} 
(-)-Depentylperhydrohistrionicotoxin (17)

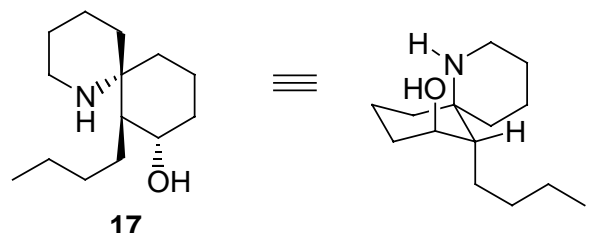

\begin{tabular}{|c|c|c|}
\hline${ }^{1} \mathrm{H} \mathrm{NMR}\left(\mathrm{CDCl}_{3}\right)$ & $\begin{array}{l}3.86(\mathrm{br} \mathrm{s}, \mathrm{W} 1 / 2=8 \mathrm{~Hz}, 1 \mathrm{H}), 3.10-2.60(\mathrm{~m}, 2 \mathrm{H}), 2.10- \\
1.0(\mathrm{~m}, 19 \mathrm{H}), 0.9(\mathrm{br} \mathrm{t}, J=6 \mathrm{~Hz}, 3 \mathrm{H})\end{array}$ & Brossi $1982^{5 \mathrm{~d}}$ \\
\hline${ }^{1} \mathrm{H} \mathrm{NMR}\left(\mathrm{CDCl}_{3}\right)$ & $\begin{array}{l}3.97(\mathrm{~m}, 1 \mathrm{H}), 3.20(\mathrm{~m}, 1 \mathrm{H}), 2.86(\mathrm{~m}, 1 \mathrm{H}), 2.1-1.0(\mathrm{~m}, \\
21 \mathrm{H}), 0.88(\mathrm{t}, J=6 \mathrm{~Hz}, 3 \mathrm{H})\end{array}$ & Pearson $1983^{\text {S1b }}$ \\
\hline${ }^{1} \mathrm{H} \mathrm{NMR}\left(\mathrm{CDCl}_{3}\right)$ & $\begin{array}{l}3.90(\mathrm{~m}, 1 \mathrm{H}), 2.95(\mathrm{~m}, 1 \mathrm{H}), 2.83(\mathrm{~m}, 1 \mathrm{H}), 2.70-2.85 \\
(\mathrm{~m}, 1 \mathrm{H}), 2.0-1.0(\mathrm{~m}, 21 \mathrm{H}), 0.90(\mathrm{t}, J=7 \mathrm{~Hz}, 3 \mathrm{H})\end{array}$ & Tanner $1986^{\mathrm{S} 1 \mathrm{c}}$ \\
\hline $\begin{array}{c}{ }^{1} \mathrm{H} \mathrm{NMR} \\
\left(\mathrm{CDCl}_{3}, \mathrm{D}_{2} \mathrm{O}, 400 \mathrm{MHz}\right)\end{array}$ & $\begin{array}{l}3.87(\mathrm{dd}, 1 \mathrm{H}), 2.92(\mathrm{~m}, 1 \mathrm{H}), 2.78(\mathrm{~m}, 1 \mathrm{H}), 1.0-2.0(\mathrm{~m}, \\
21 \mathrm{H}), 0.90(\mathrm{t}, J=7 \mathrm{~Hz}, 3 \mathrm{H})\end{array}$ & Carruthers $1983^{\text {S1d }}$ \\
\hline $\begin{array}{c}{ }^{13} \mathrm{C} \mathrm{NMR} \\
\left(\mathrm{CDCl}_{3}, \mathrm{D}_{2} \mathrm{O}, 400 \mathrm{MHz}\right)\end{array}$ & $\begin{array}{l}69.9,54.6,41.9,40.2,37.2,33.3,30.4,28.0,27.2(2 \mathrm{C}) \text {, } \\
22.9,19.4,15.1,13.9\end{array}$ & Carruthers $1983^{\text {Sld }}$ \\
\hline $\begin{array}{c}{ }^{1} \mathrm{H} \mathrm{NMR} \\
\left(\mathrm{CDCl}_{3}, \mathrm{D}_{2} \mathrm{O}, 300 \mathrm{MHz}\right)\end{array}$ & $\begin{array}{l}3.87(\mathrm{dd}, J=5.4,2.7 \mathrm{~Hz}, 1 \mathrm{H}), 2.92(\mathrm{~m}, 1 \mathrm{H}), 2.78(\mathrm{~m}, \\
1 \mathrm{H}), 1.0-2.0(\mathrm{~m}, 21 \mathrm{H}), 0.89(\mathrm{t}, J=6.9 \mathrm{~Hz}, 3 \mathrm{H})\end{array}$ & $\begin{array}{l}\text { Our synthetic } \\
\text { compound }\end{array}$ \\
\hline $\begin{array}{c}{ }^{13} \mathrm{C} \mathrm{NMR} \\
\left(\mathrm{CDCl}_{3}, \mathrm{D}_{2} \mathrm{O}, 300 \mathrm{MHz}\right)\end{array}$ & $\begin{array}{l}69.9,54.5,41.8,40.1,37.2,33.2,30.5,27.9,27.3,27.2, \\
23.0,19.5,15.1,14.0\end{array}$ & $\begin{array}{l}\text { Our synthetic } \\
\text { compound }\end{array}$ \\
\hline
\end{tabular}




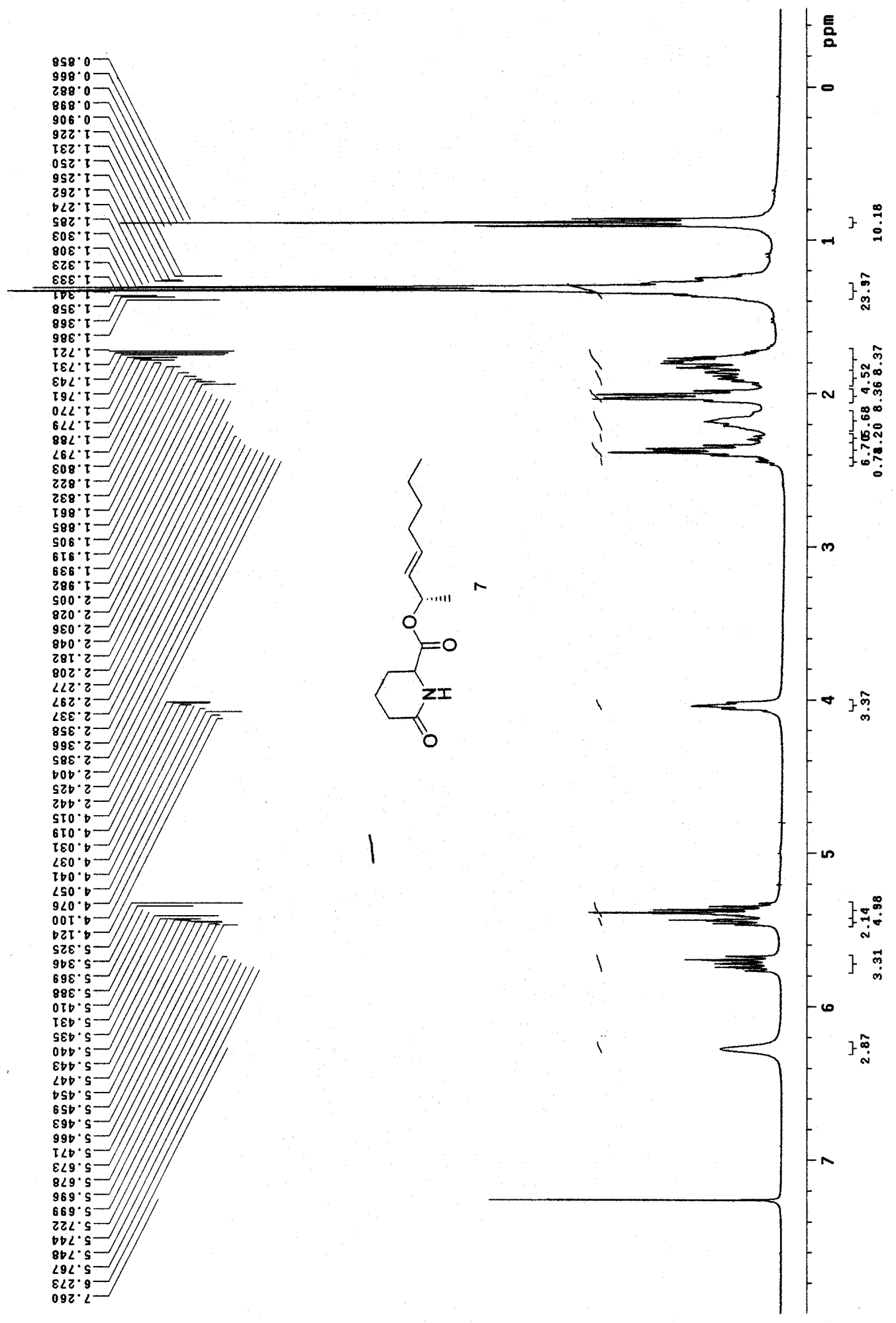




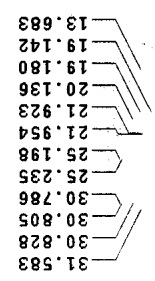

$059 \cdot \mathrm{DS}=$
$\mathrm{D} 89 \cdot \mathrm{DS}-$

$869 \cdot 2 L$

$962 \cdot 2 L-1$

$000.2 L-\square$

$s 20 \cdot 2 L$

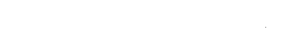

SID. $82 I \longrightarrow$

$205.82 t$

$99 Z^{\circ} b \varepsilon I$
S॰ $\varepsilon^{\circ} b \varepsilon \tau$

$\angle b Z^{\circ} 0 \angle \tau$

$\varepsilon 9 \varepsilon^{\circ} \cdot \tau<\tau$
$\varepsilon 6 \varepsilon \cdot \tau<\tau$

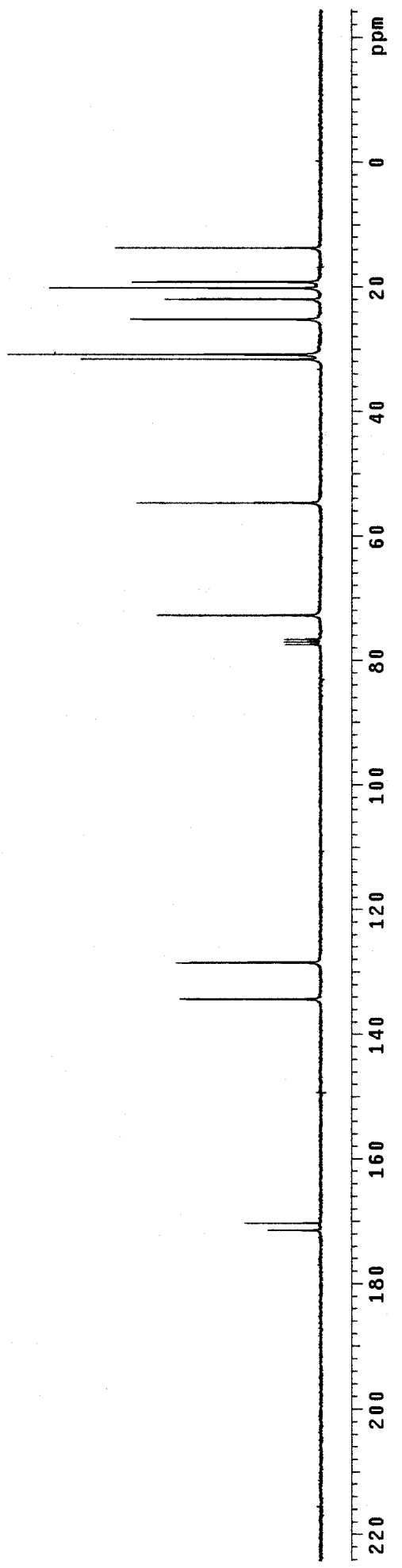




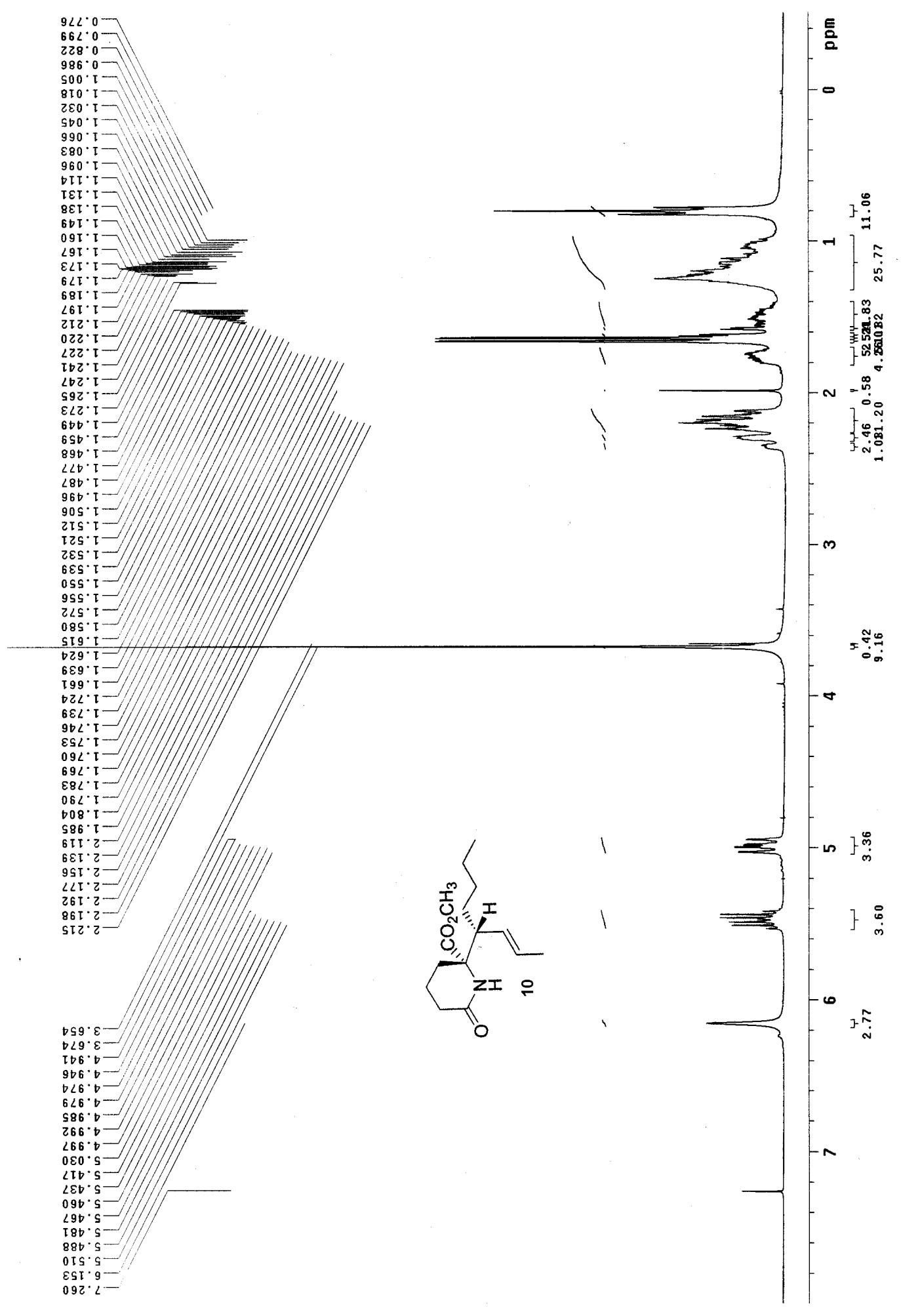



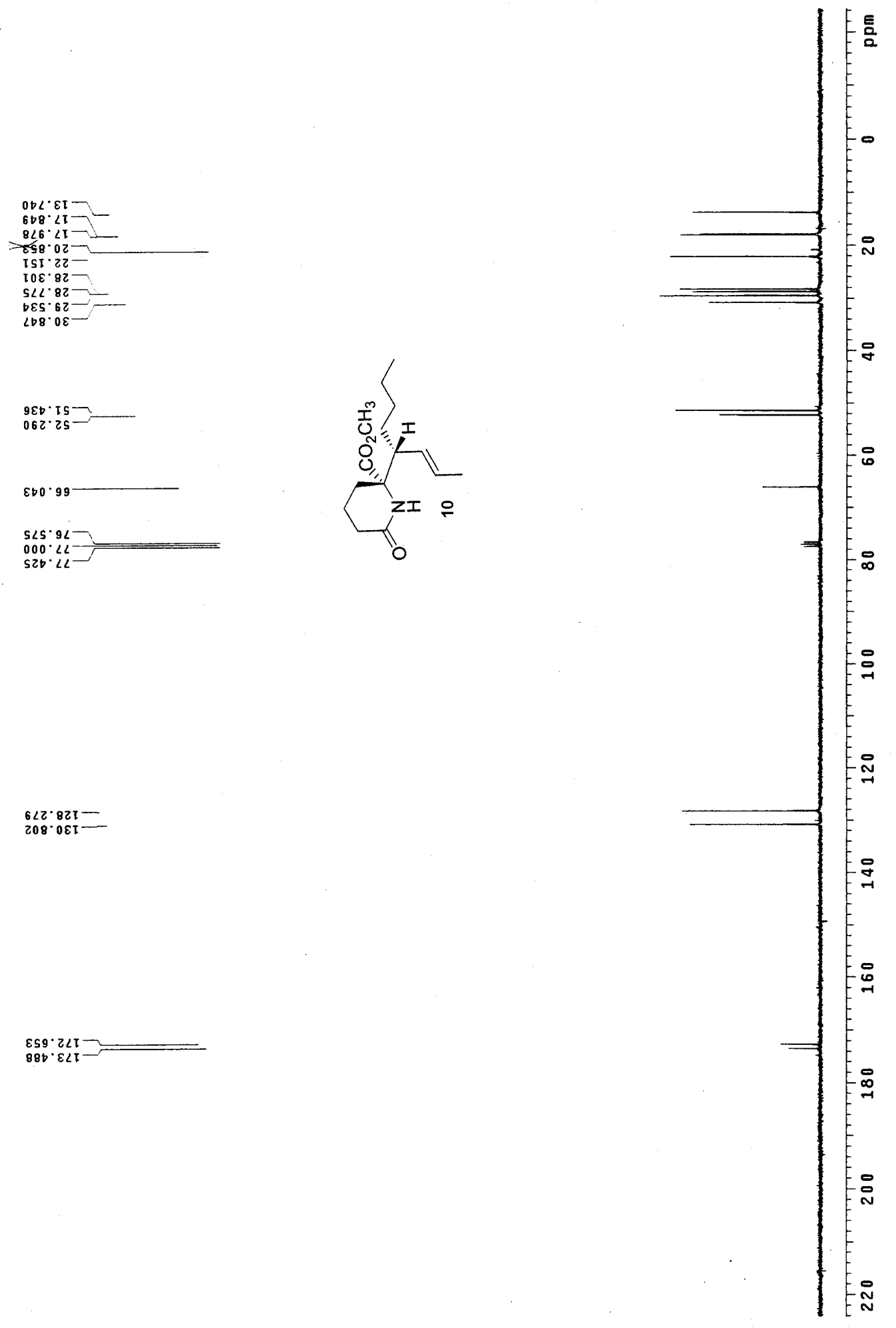


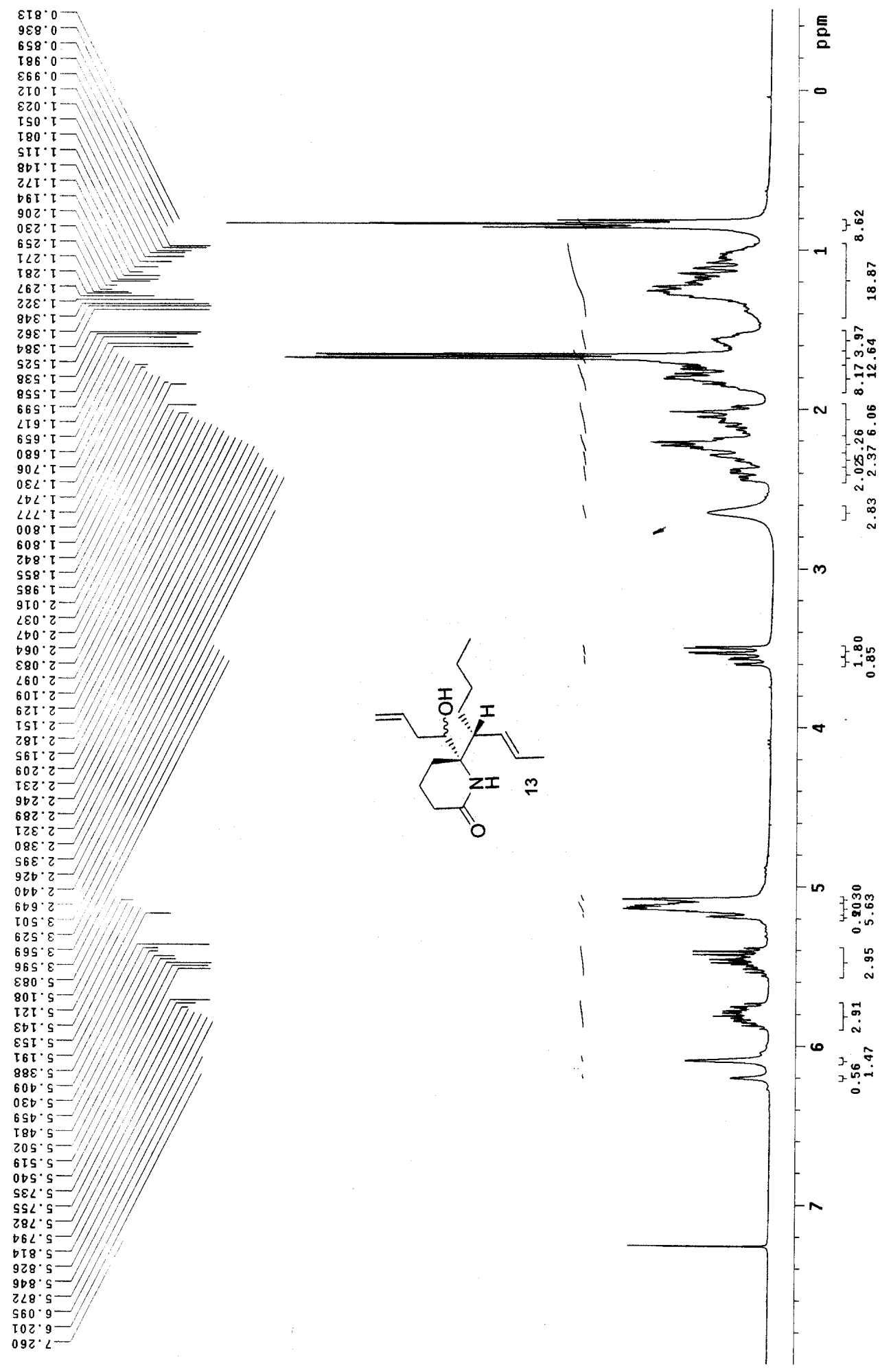



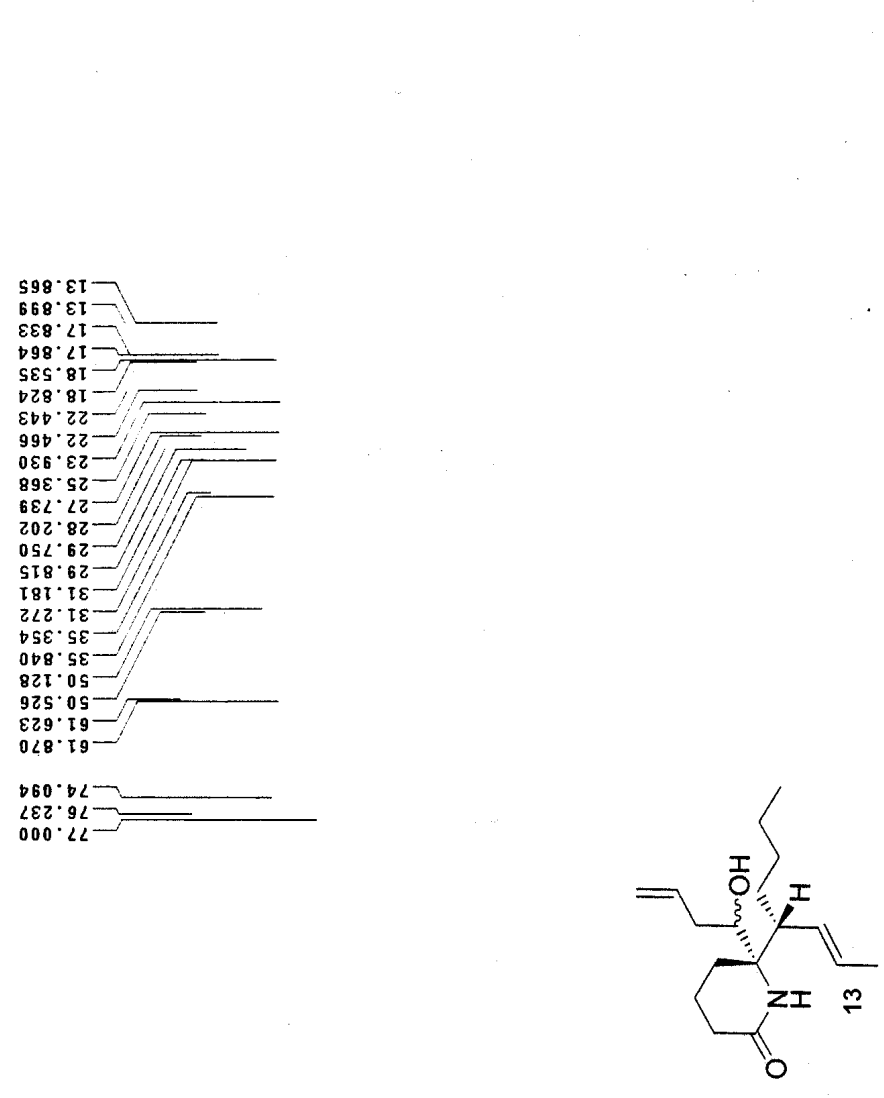

SST. $\angle I T-$

$200 \cdot \angle I I$

$062.0 \varepsilon I$

$\varepsilon b \varepsilon^{\circ} \varsigma \varepsilon \mathrm{I}$
$299 \cdot 5 \varepsilon \mathrm{I}-$

$\tau \angle 8^{\circ} \varepsilon \angle \tau=$
$6 S D^{\circ} b<\tau$

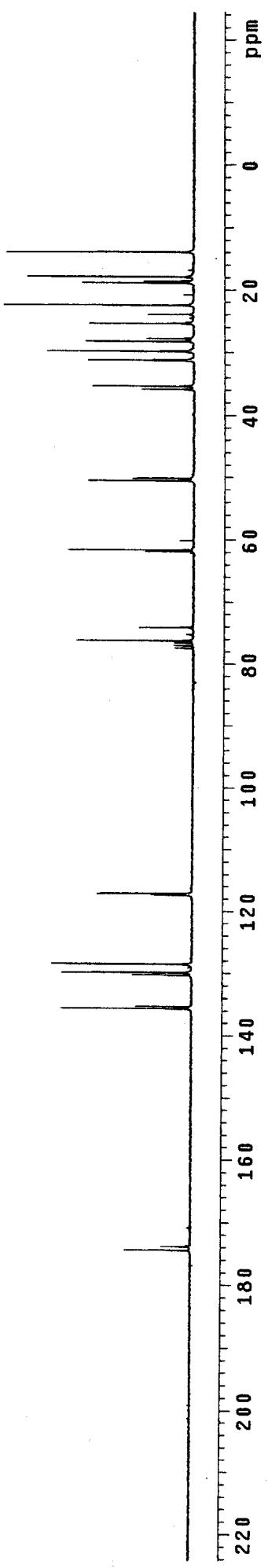




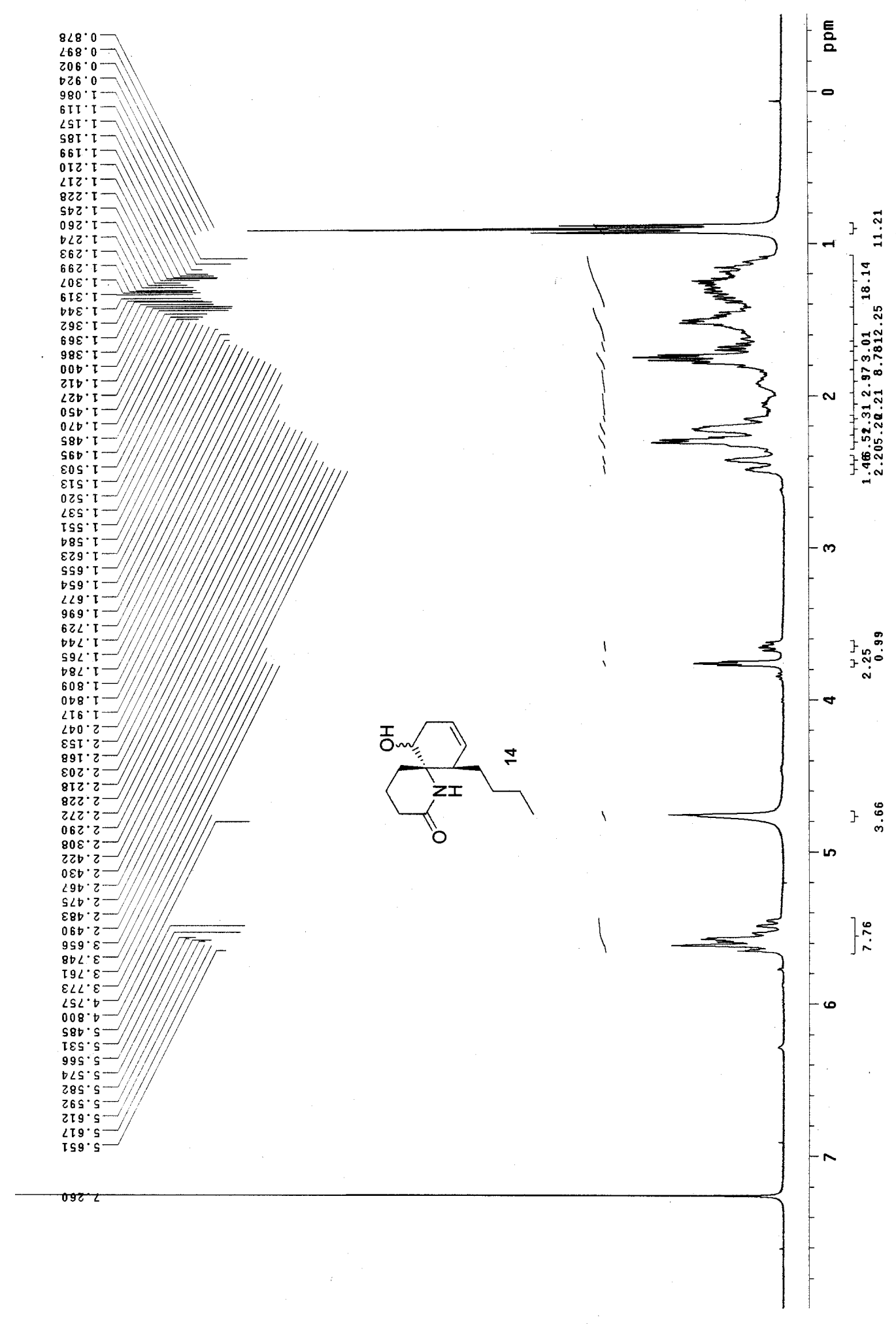



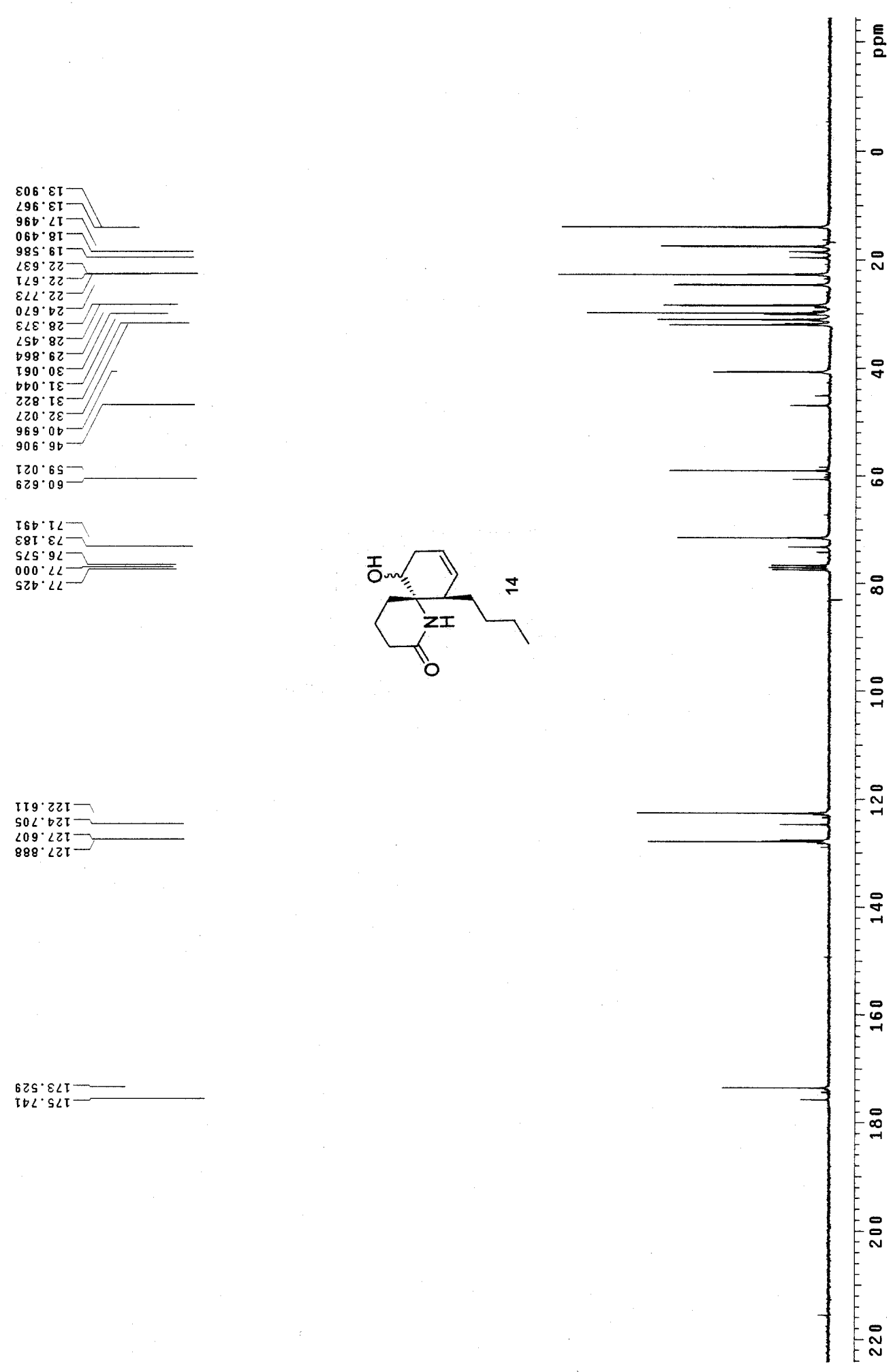


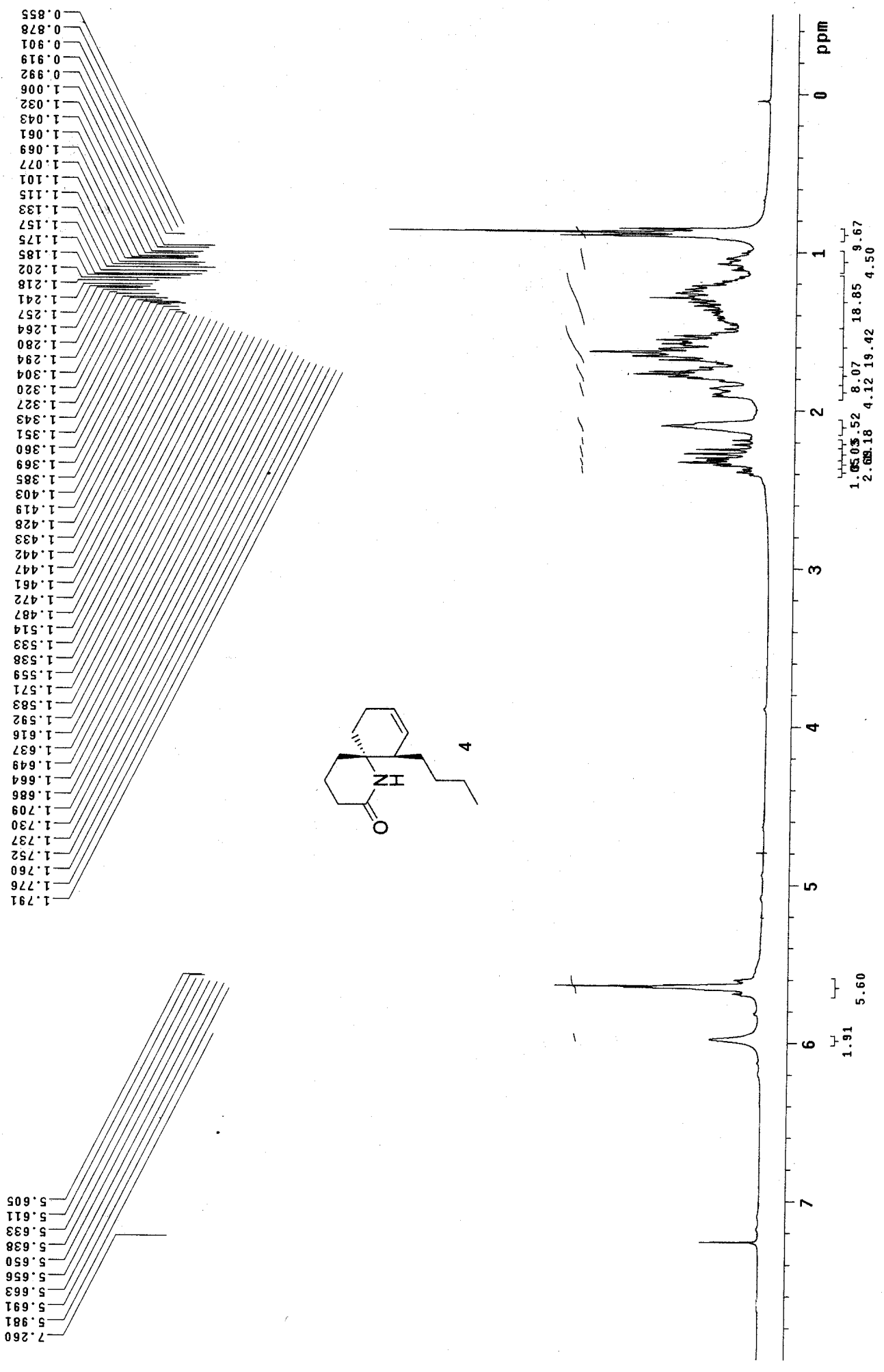




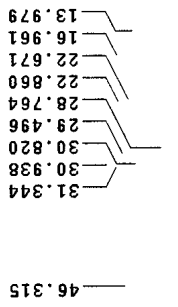

I6S.sS

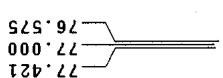

000.24

I2b 22

$060 \cdot 921=$
$517 \cdot 821=$

$022 \cdot 2 \angle I$

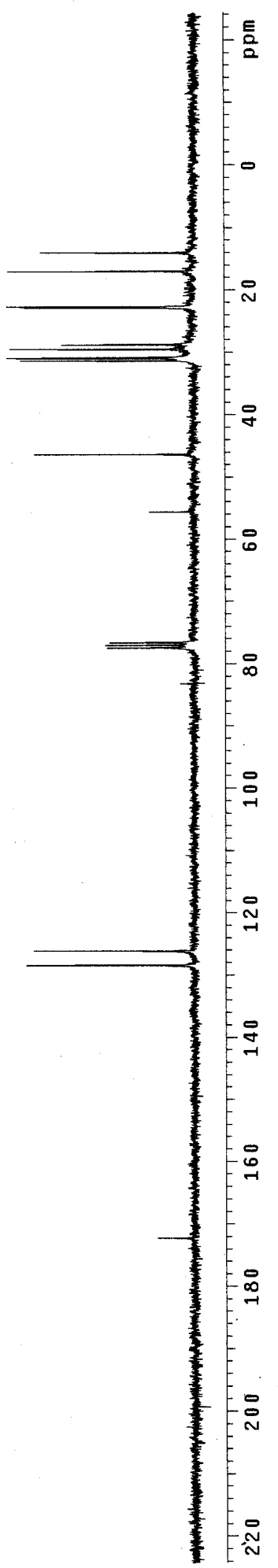




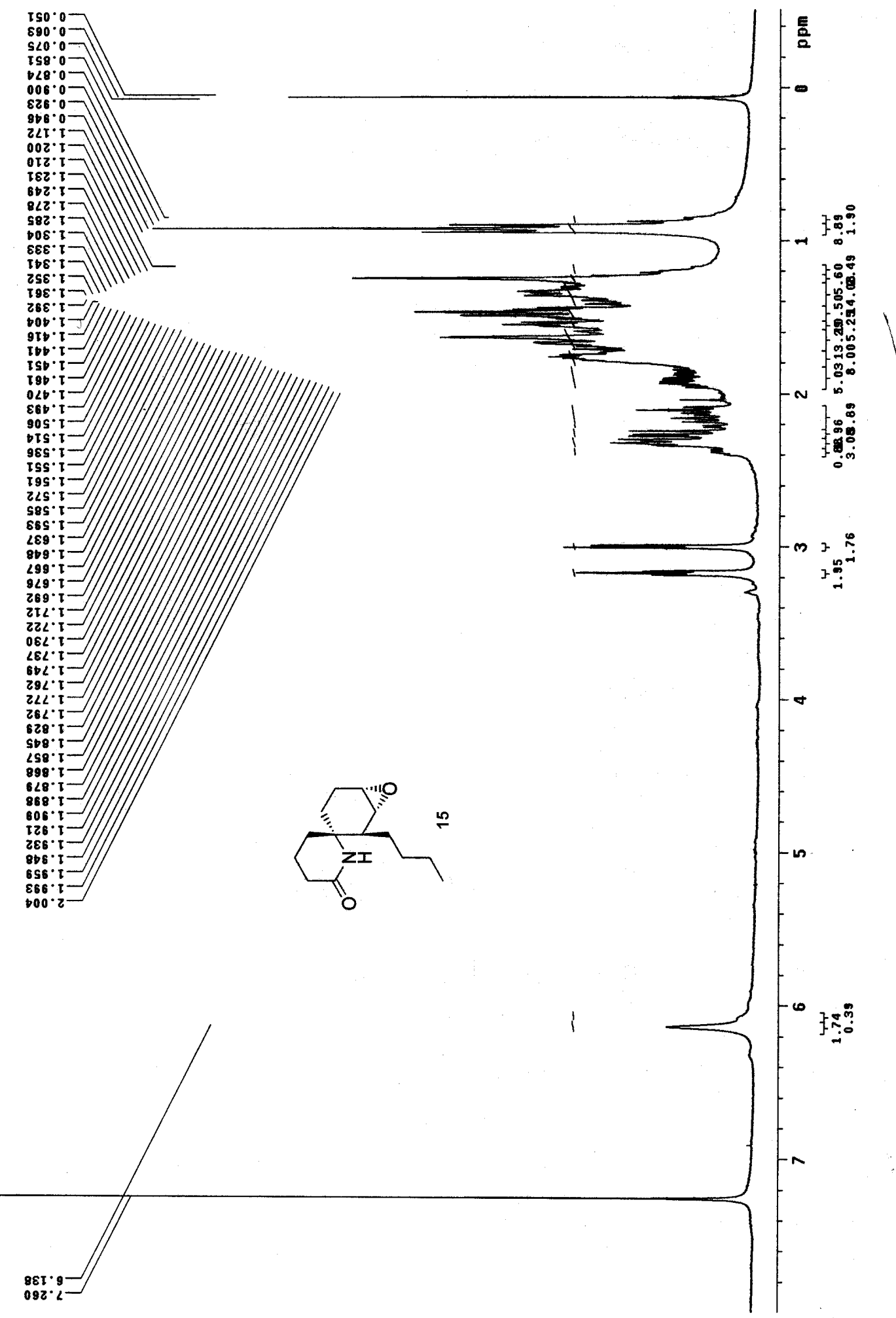



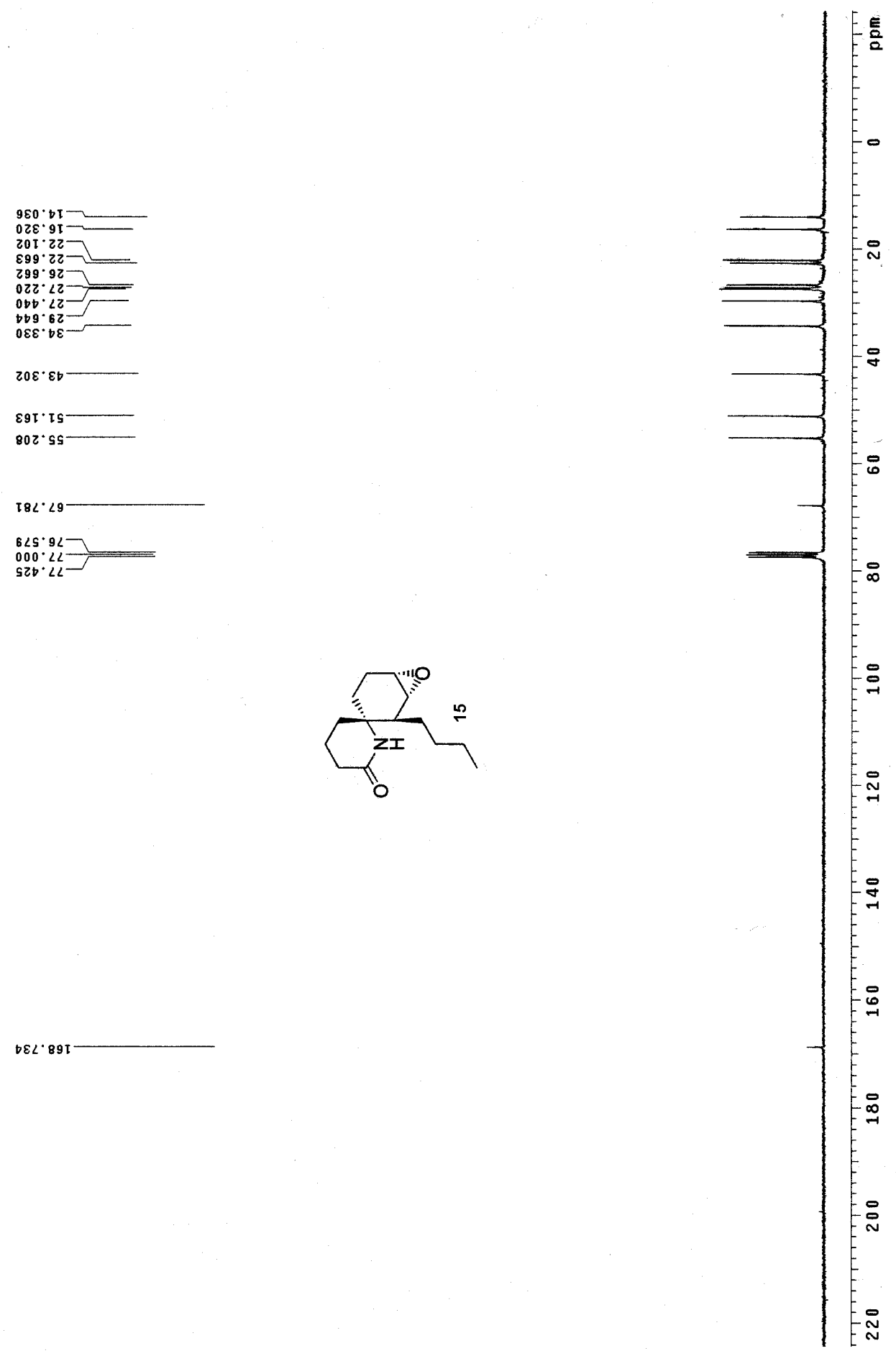


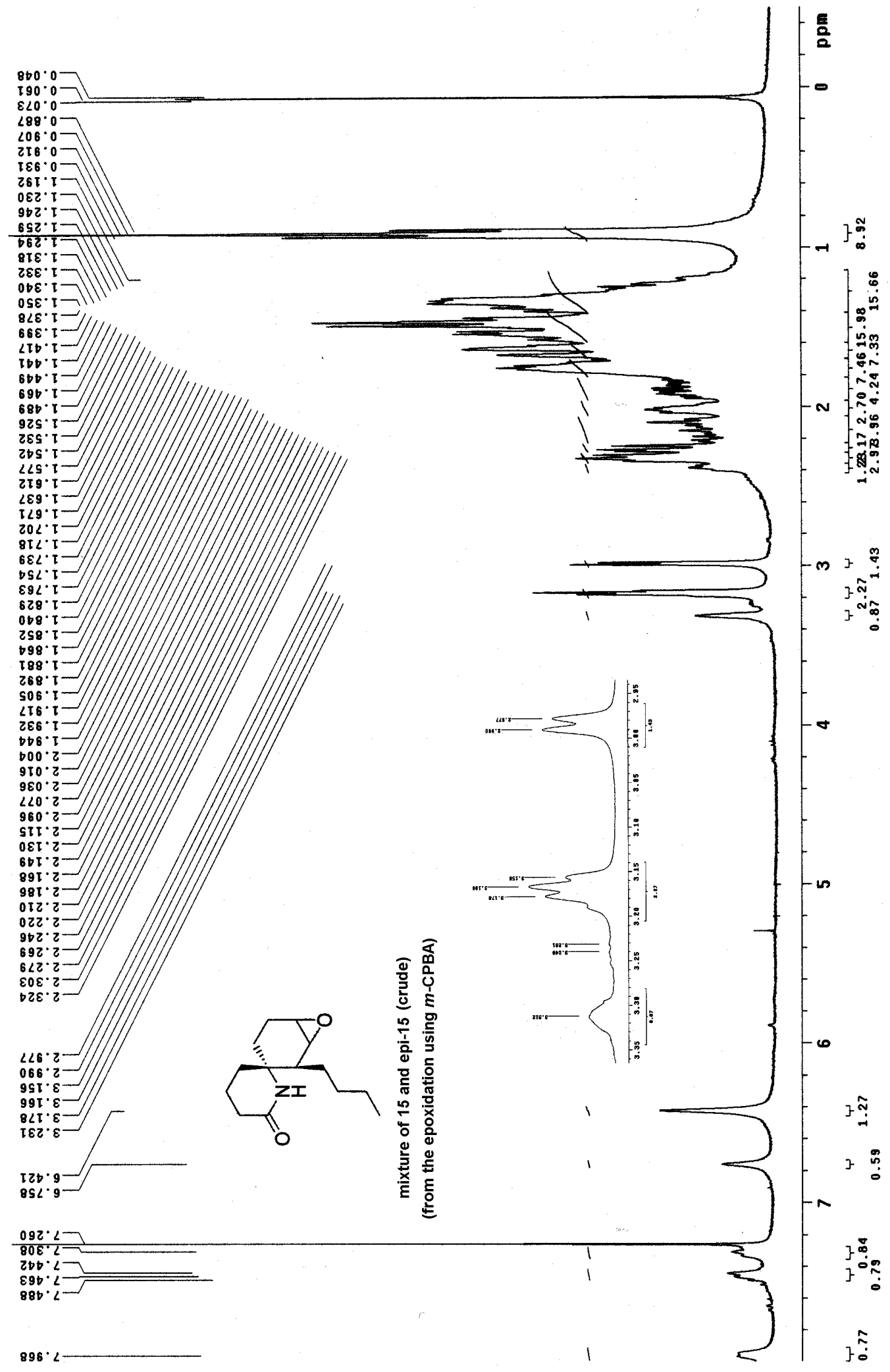



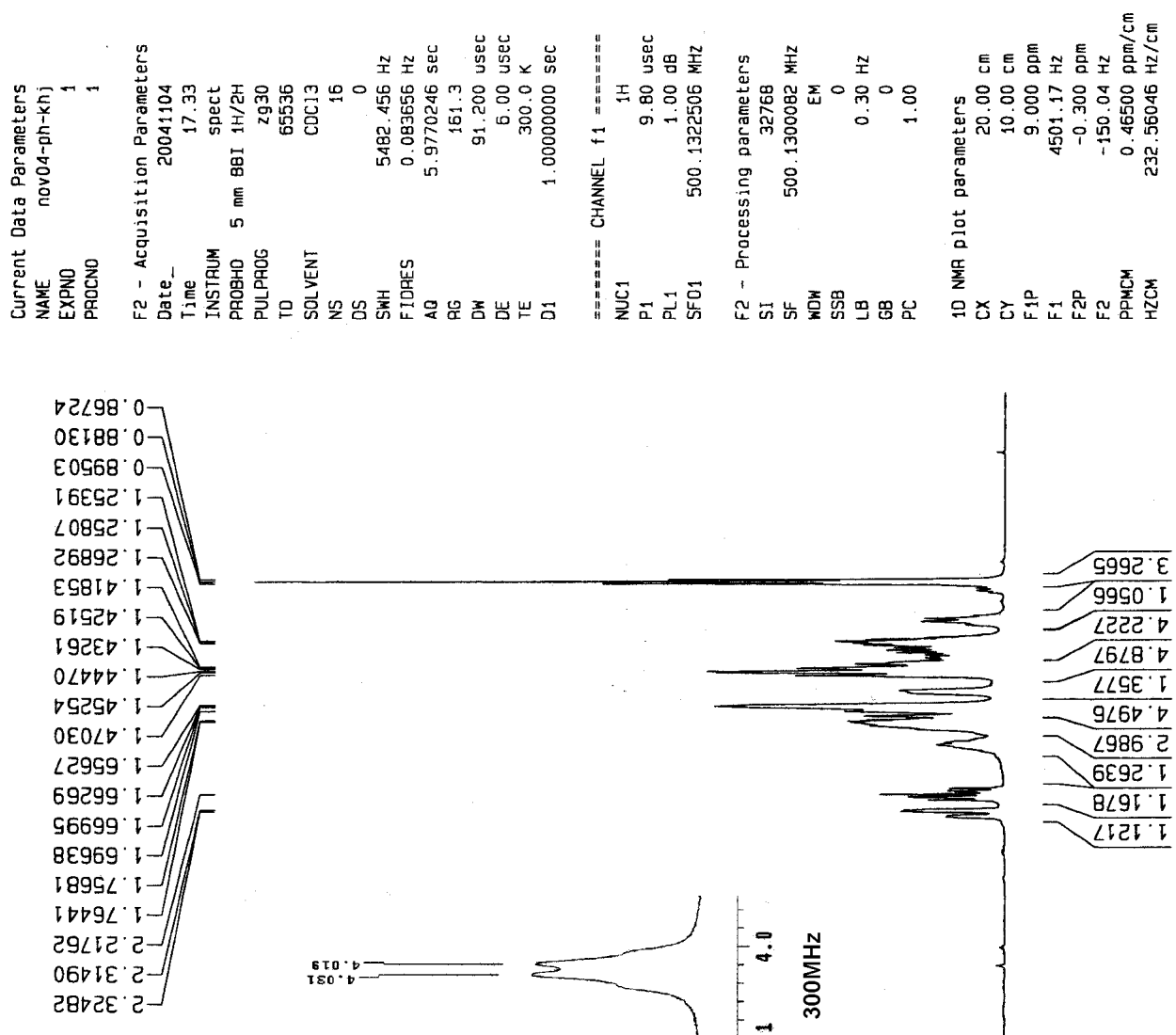

$29000^{\circ} \circ \longrightarrow$

$E \angle S D O O^{\circ} \longrightarrow$

I6989 $\triangleright$

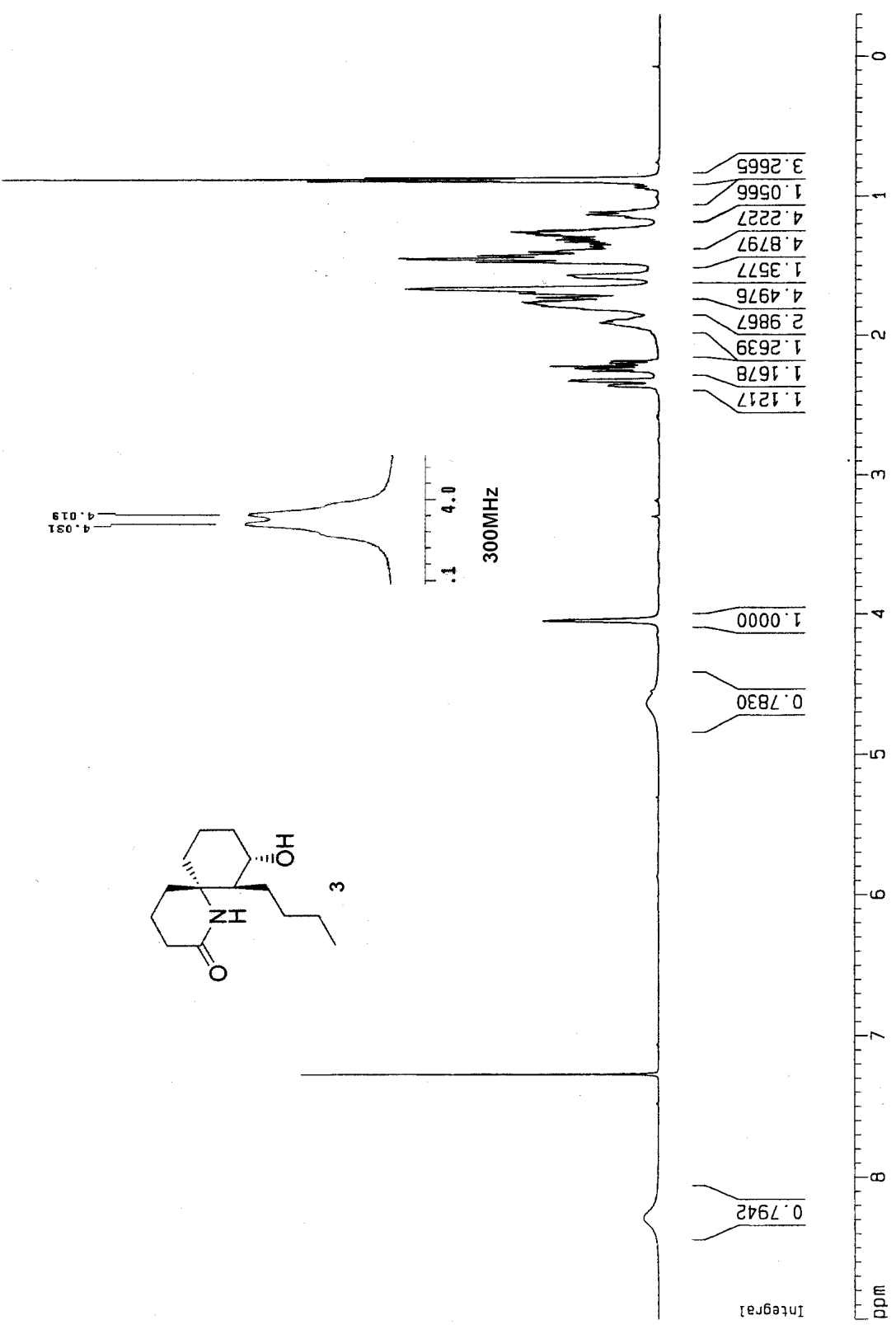

wdd 

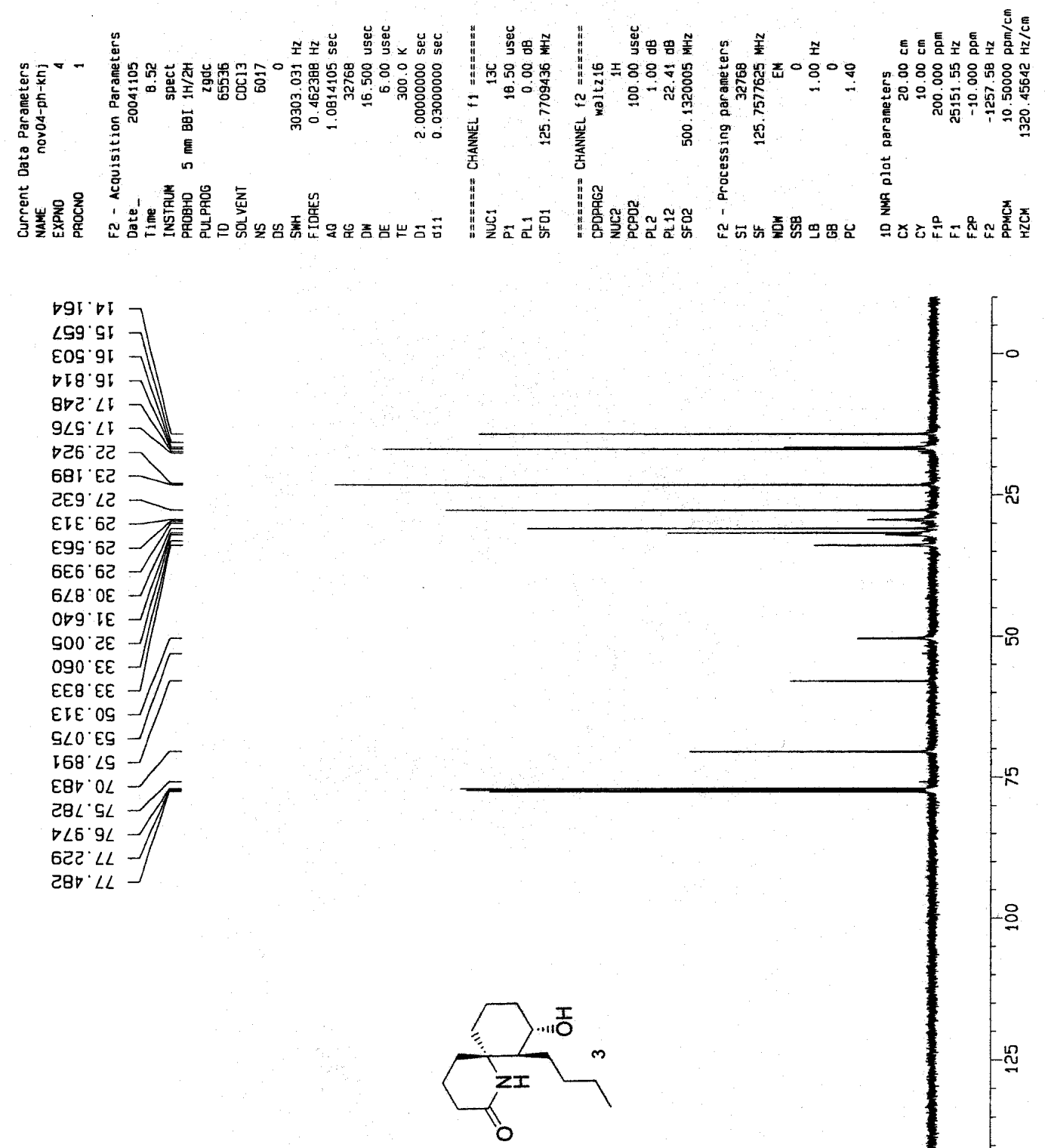

$810^{\circ} 2 \angle t$

udd

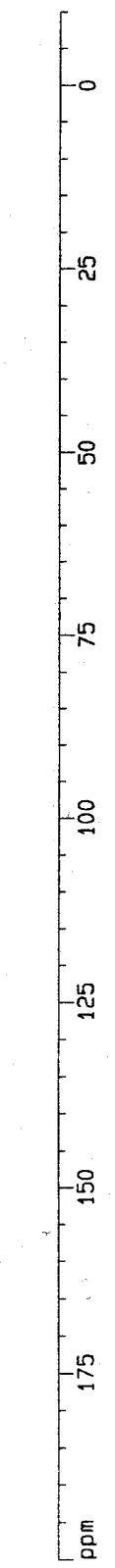




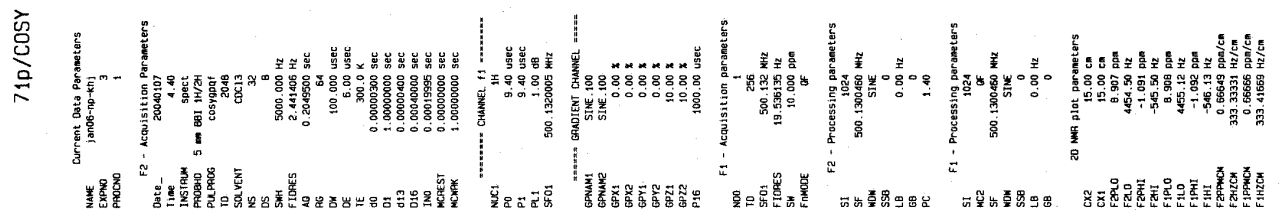
$\underbrace{\sum_{2 I}^{I}}_{2 I}$

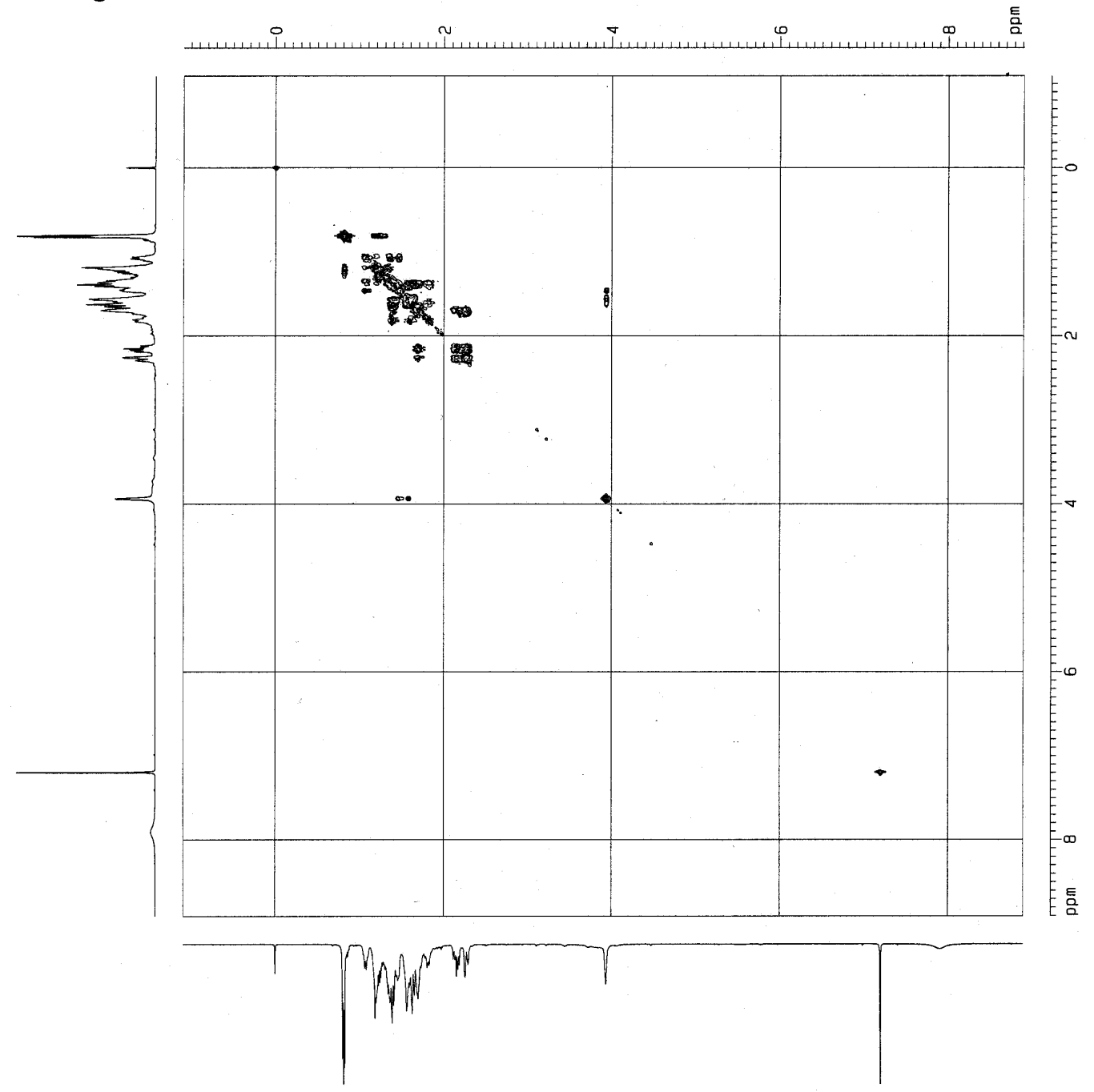



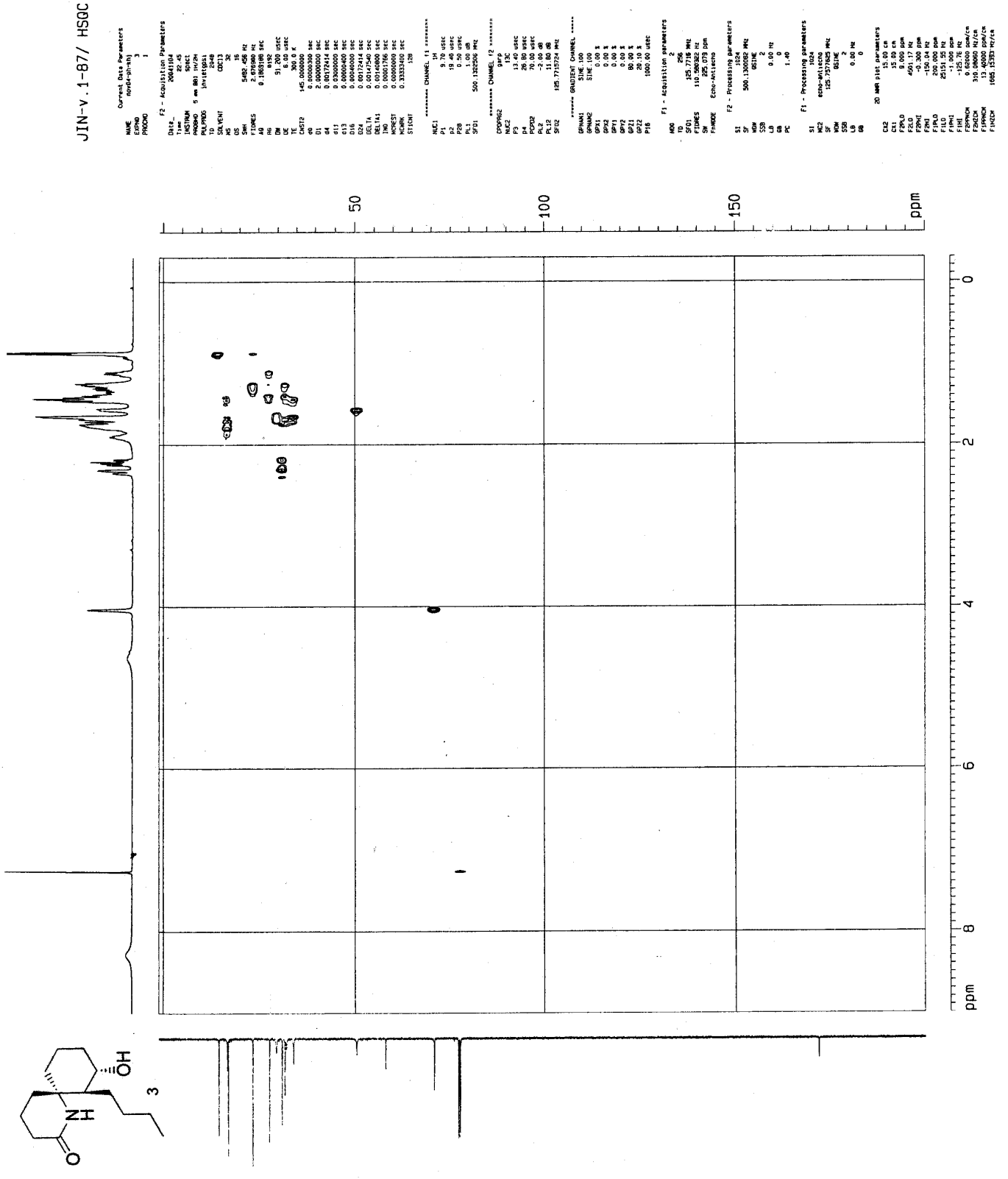


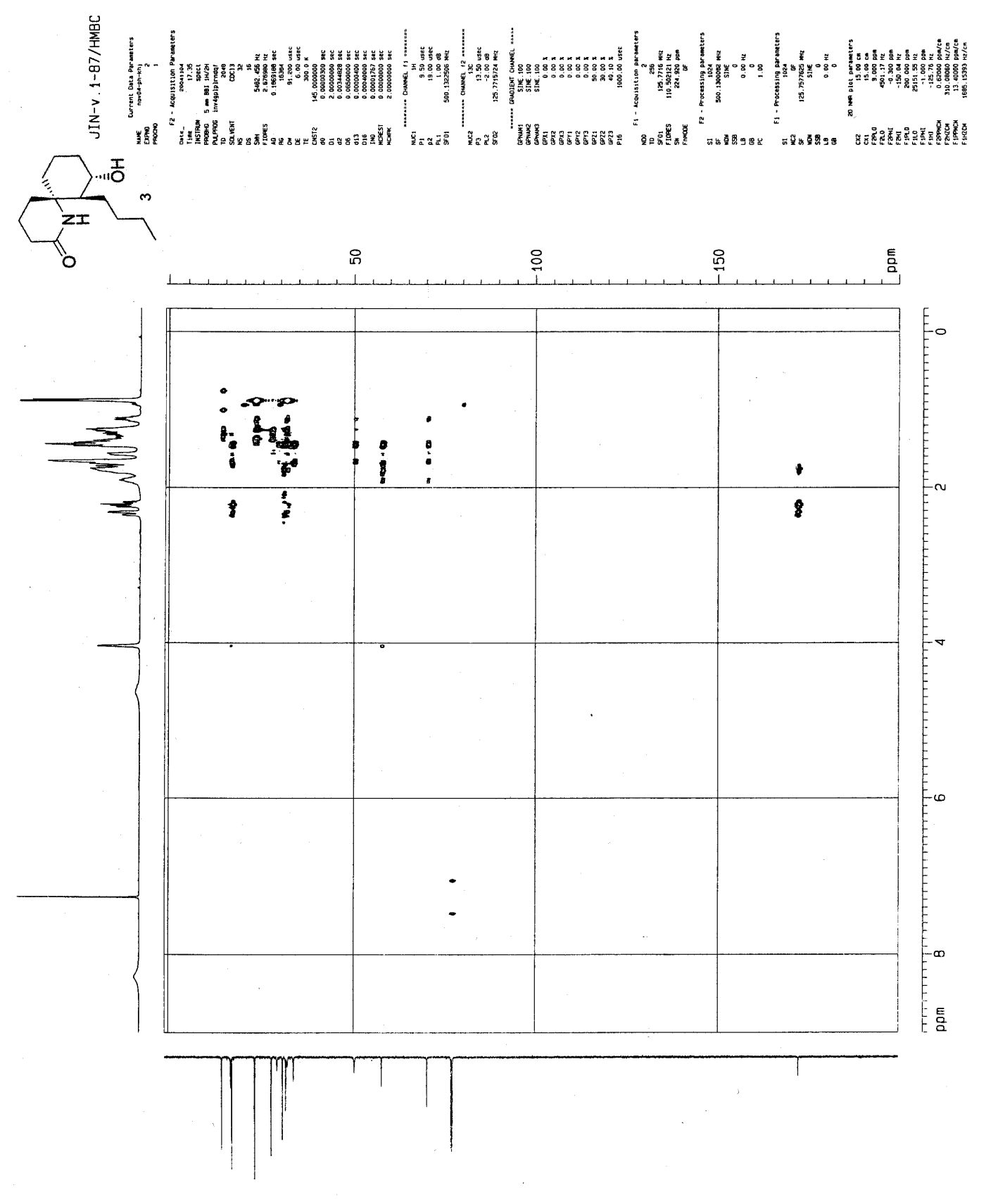




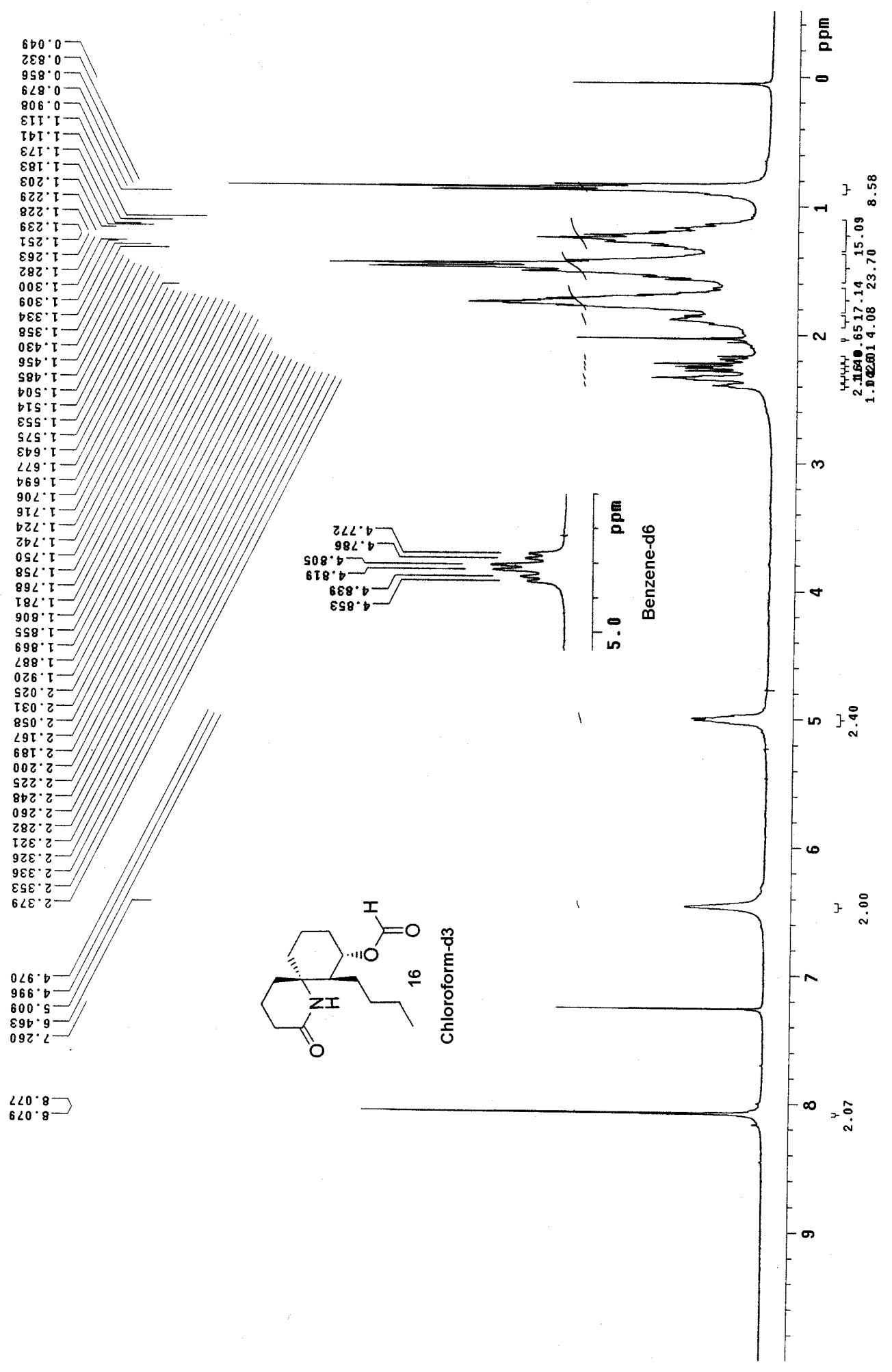



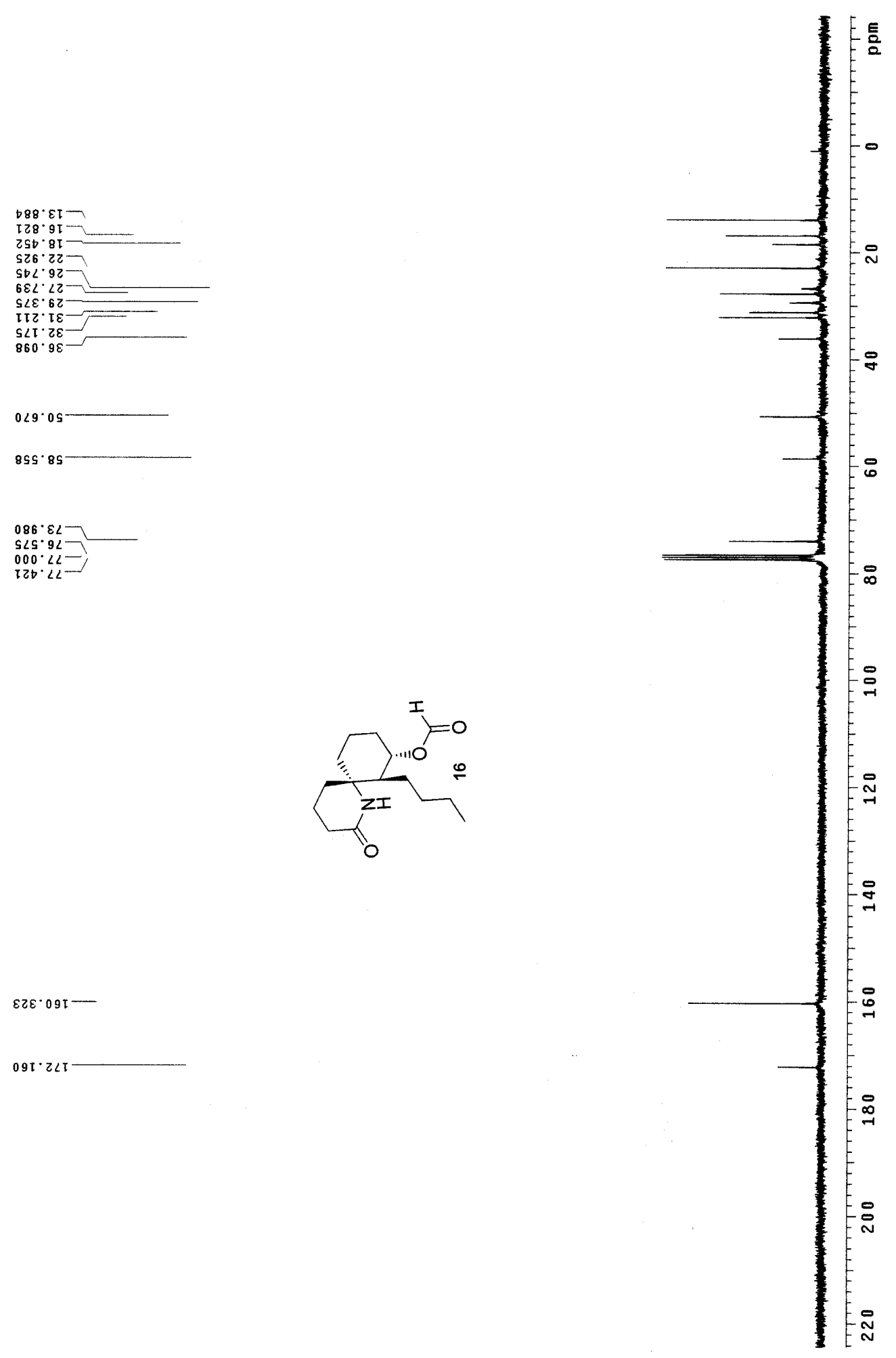


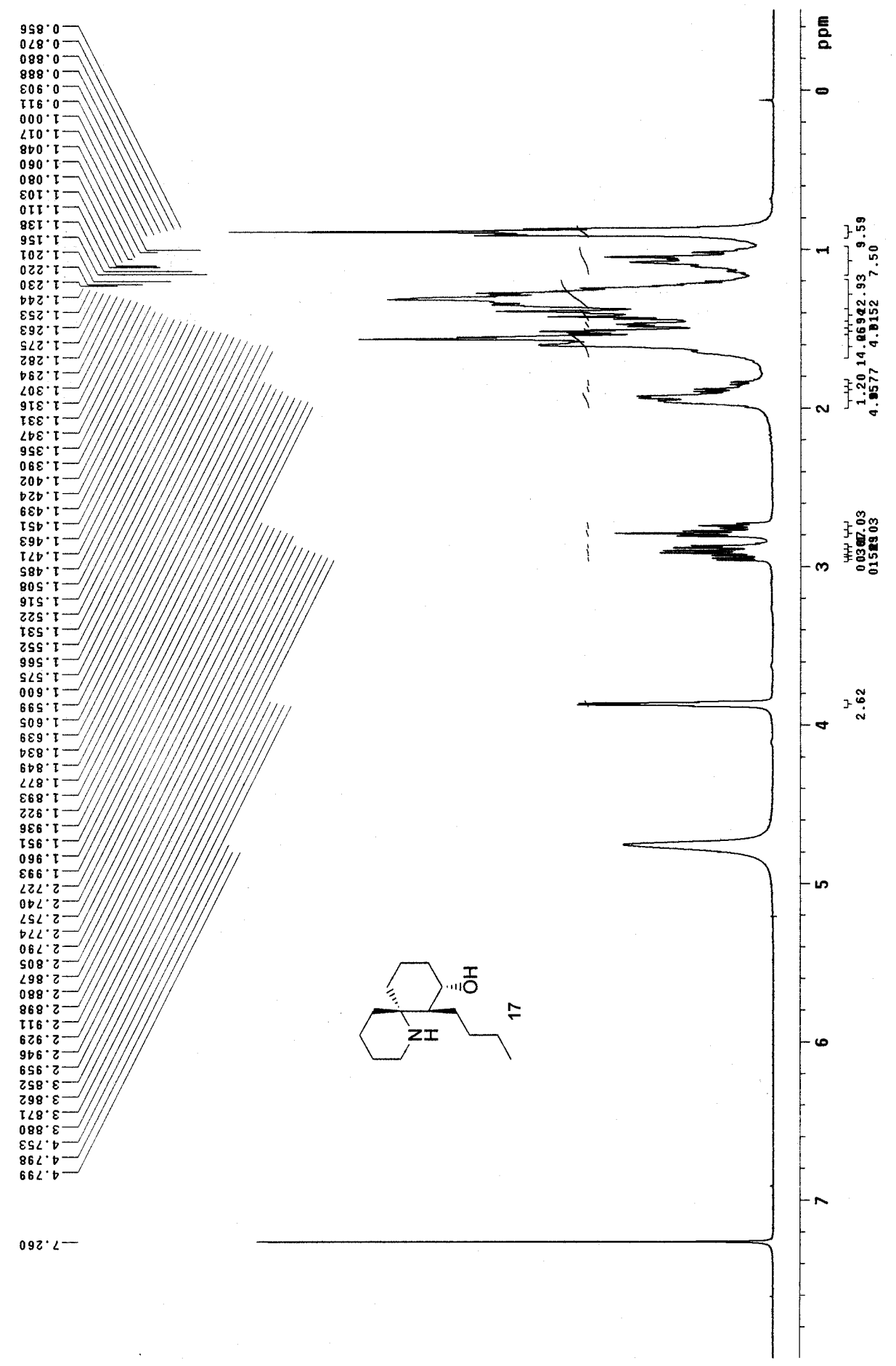



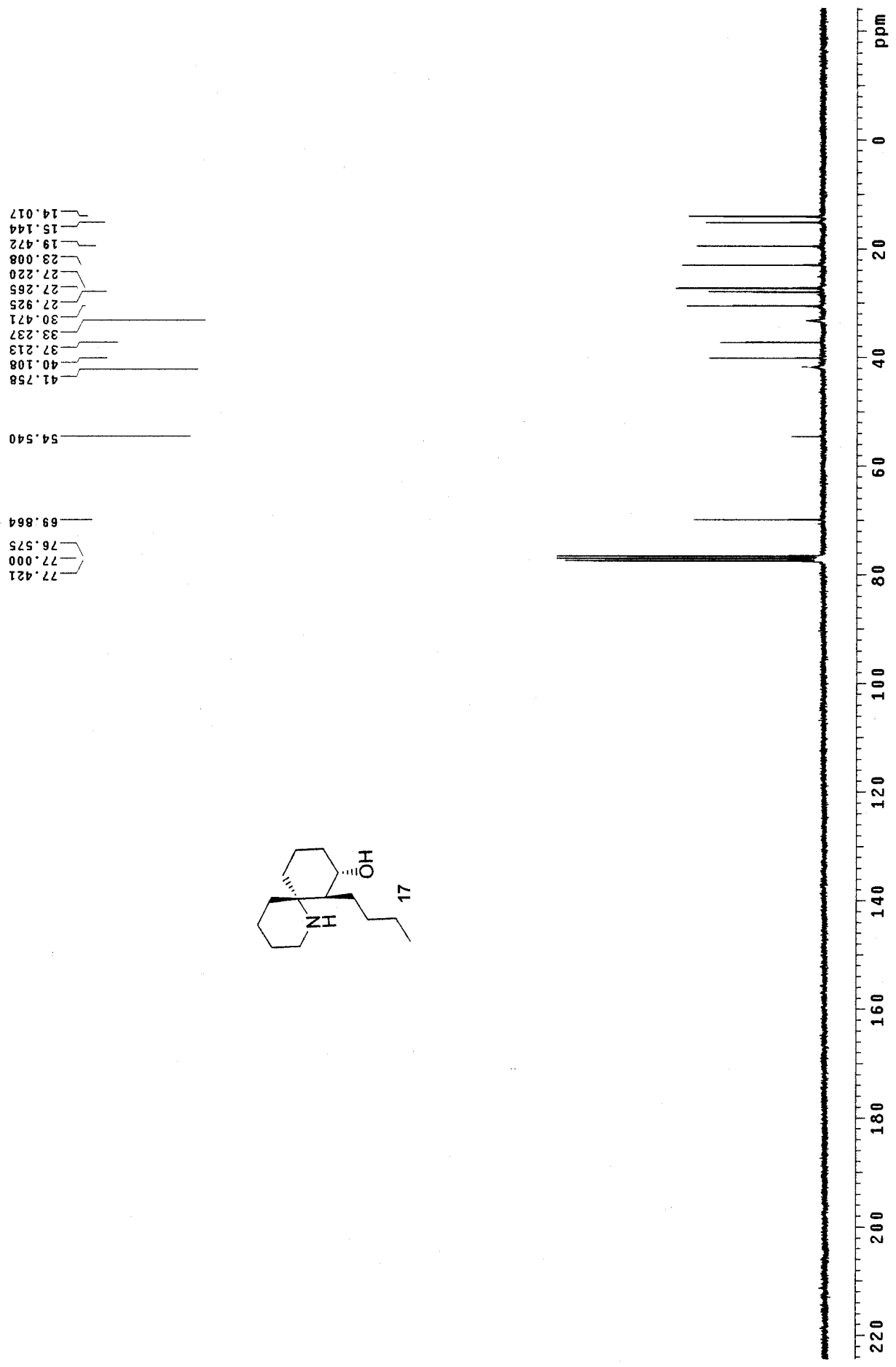